Please do not remove this page

RMIT

UNIVERSITY

\title{
Cascaded multicavity resonantly enhanced Mach-Zehnder modulator
}

Nguyen, Giang; Visagathilagar, Yuvaraja; Mitchell, Arnan

https://researchrepository.rmit.edu.au/esploro/outputs/9921862895101341/filesAndLinks?institution=61RMIT_INST\&index=null

Nguyen, G., Visagathilagar, Y., \& Mitchell, A. (2006). Cascaded multicavity resonantly enhanced Mach-Zehnder modulator. Proceedings of the 31st Australian Conference on Optical Fibre Technology and Australian Optical Society (ACOFT\&AOS 2006), 28-30.

https://researchrepository.rmit.edu.au/discovery/fulldisplay/alma9921862895101341/61RMIT_INST:Resea rchRepository

Repository homepage: https://researchrepository.rmit.edu.au

(c) 2006 IEEE. Personal use of this material is permitted. However, permission to reprint/republish this material for advertising or promotional purposes or for creating new collective works for resale or redistribution to servers or lists, or to reuse any copyrighted component of this work in other works must be obtained from the IEEE.

Downloaded On 2023/04/26 11:27:40 +1000 


\title{
Cascaded Multicavity Resonantly Enhanced Mach-Zehnder Modulator
}

\author{
Thach G. Nguyen, Yuvaraja S. Visagathilagar, and Arnan Mitchell \\ School of Electrical and Computer Engineering, RMIT University, GPO Box 2476V, \\ Melbourne, Victoria 3001, Australia
}

\begin{abstract}
A resonantly enhanced modulator structure with cascaded multiple resonant cavities is proposed and analyzed. The microwave effective index of the electrode can be much higher than the optical index. Therefore, the electrode structure can be optimized for high modulation efficiency and high resonant enhancement.
\end{abstract}

\section{INTRODUCTION}

Mach-Zehnder optical modulators (MZMs) on $\mathrm{LiNbO}_{3}$ have been widely used in optical communication systems. Conventional modulators which employ a travelling-wave electrode structure offer very large bandwidth but low modulation efficiency. Therefore, the RF links that use MZMs suffer large link loss. In band-operation applications such as radio-on-fiber [1] and local oscillator distribution for photonic mixing [2], only a narrow bandwidth around the center frequency is required. Hence, resonant electrode structures [3], [4] can be employed in MZMs to improve the modulation efficiency at the expense of modulation bandwidth.

In order to achieve high modulation efficiency with resonant enhancement, the modulator should have low inherent switching voltage and high resonant enhancement. Resonant enhancement depends strongly on the loss of the electrode. We have practically demonstrated a highly efficient resonantly enhanced modulator (RE-MZM) on $\mathrm{X}$-cut $\mathrm{LiNbO}_{3}$ [5]. The electrode structure of this REMZM was numerically optimized [6] to provide low inherent switching voltage and low RF loss. However, the operating frequency was limited to less than $2 \mathrm{GHz}$. In the literature, there are several demonstration of REMZMs at frequencies higher than $10 \mathrm{GHz}$ [4], [7]. These modulators had very high modulation enhancement with very short electrodes. Because of very short electrodes, the overall modulation efficiency of these RE-MZMs is quite low.

To increase the overall modulation efficiency, the electrode length should be made large. However, at high frequencies, in order to maintain a long electrode, the microwave effective index of the electrode must be matched to the effective index of the optical waveguide. For $\mathrm{LiNbO}_{3}$ modulators, index matched electrodes usually have low inherent modulation efficiency and high RF loss. Furthermore, the total RF loss of a long electrode is very high. Therefore, RE-MZMs with long electrodes may not provide modulation efficiency improvement over travelling-wave modulators. To overcome the limitations of long electrode RE-MZMs, we proposed cascaded multicavity RE-MZM [8].

This paper presents the analysis of the proposed REMZM structure. Particular emphasis is placed on the effectiveness of multicavity approach in improving modulation efficiency with long electrode through resonant enhancement.

\section{CASCADED MULTiCAVITY RE-MZM}

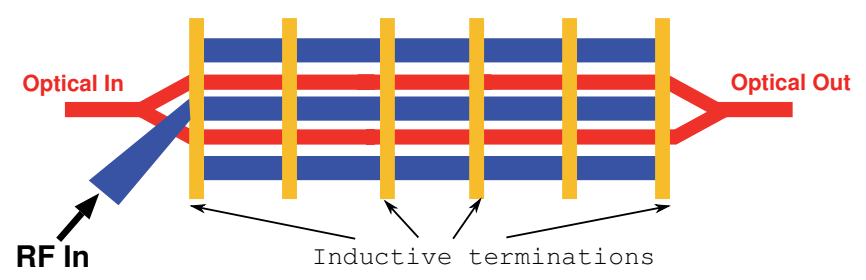

Fig. 1. Cascaded multicavity RE-MZM structure.

In Fabry-Perot type RE-MZMs [9], the lengths of the resonant cavities are slightly less than a multiple of half of wavelength. At high frequencies, the wavelengths of the microwave signals are very short. Thus, it is possible to divide a long electrode into a number of resonant cavities [8]. Fig. 1 presents the structures of a cascaded multicavity RE-MZM. Inductive terminations are periodically added to the RF electrode to form a structure with cascaded Fabry-Perot resonant cavities. Within each cavity, strong field enhancement can be achieved provided RF loss is low. The RF power is coupled from the previous cavity into the next cavity through the inductive terminations.

In the structure of Fig. 1, the length of each resonant cavity is much shorter than the whole electrode length, therefore, the microwave effective of the electrode can be much higher than the optical index without detrimental effect to the modulation efficiency of each resonant cavity. Furthermore, the RF loss of each resonant cavity is reduced due to short length. As a consequence, the resonant enhancement factor of each cavity is improved.

With inductivel terminations, the inductance of the loaded transmission line is lower than that of the unloaded electrode. Hence, the average microwave effective index of the inductively loaded electrode is lower than the effective index of the original electrode. By careful design of the inductive terminations, phase matching between the standing electric wave and the lightwave can 


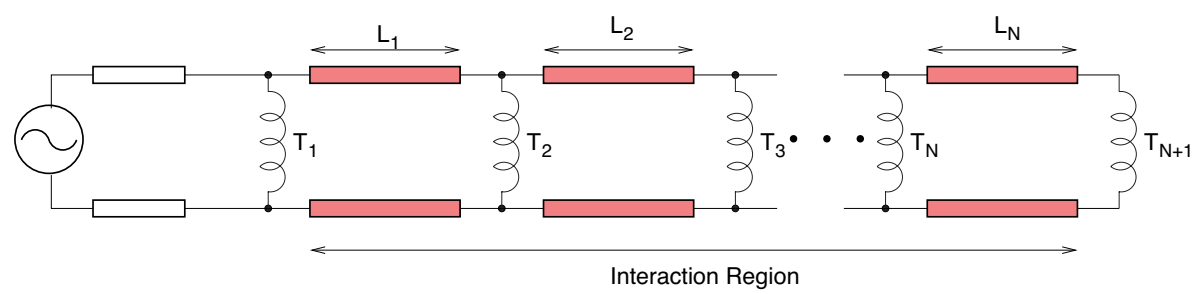

Fig. 2. Equivalent circuit model of a cascaded multicavity RE-MZM.

be achieved. Therefore, the constraint of index matching which usually comes with long electrode travelling-wave modulator can be removed. The electrode structure can be optimized for high inherent modulation efficiency and high resonant enhancement. In the following sections, the effectiveness of this cascaded RE-MZM structure will be investigated.

\section{NUMBER OF RESONANT CAVITIES AND THE MODULATION EFFICIENCY}

In this Section, the relationship between the number of resonant cavities and the performance of a cascaded multicavity RE-MZM is analyzed. Similar to [6], the inductive terminations are simply modelled as ideal inductive elements in this analysis. Fig. 2 illustrates the equivalent circuit model of a cascaded multicavity REMZM. For simplification of investigation, all cavities have the electrode length and all inductive terminations are identical except the first and last ones. A CPW electrode structure on $\mathrm{X}$-cut $\mathrm{LiNbO}_{3}$ is considered. As investigated in [6], the electrode has a Gap-Width-Gap configuration of 50-16-50 $\mu \mathrm{m}$, electrode thickness of $30 \mu \mathrm{m}$ and buffer layer thickness of $0.2 \mu \mathrm{m}$ [6]. This electrode configuration provides the best trade-offs between the resonant enhancement and inherent switching voltage. The characteristics of the electrode obtained from a full-wave 2-D FEM simulator [10] are RF attenuation constant $\alpha_{c}=0.192 \mathrm{~dB} / \mathrm{cm} \sqrt{\mathrm{GHz}}$, microwave effective index $N_{m}=3.086$, characteristic impedance $Z_{c}=37.6 \Omega$ and inherent switching voltage length product $V_{\pi} L=$ $9.33 \mathrm{Vcm}$. The microwave effective index of the electrode is much higher than the effective index of the guided optical mode which is assumed to be $N_{o}=2.15$. A numerical optimizer similar to the one described in [6] was employed to search for the length of each resonant cavity and inductive elements to maximize the overall modulation efficiency with a given number of cavities. An upper limit of $4 \mathrm{~cm}$ was imposed on the total electrode length. The optimization was conducted at a single frequency of $20 \mathrm{GHz}$. The responses of the RE-MZMs were simulated by a numerical network model [11].

Fig. 3 shows the length of each resonant cavity of RE-MZMs with different number of resonant cavities. It can be seen that the electrode length of the single cavity RE-MZM is only $4.3 \mathrm{~mm}$ which is much shorter than the maximum allowed length of $4 \mathrm{~cm}$. This very

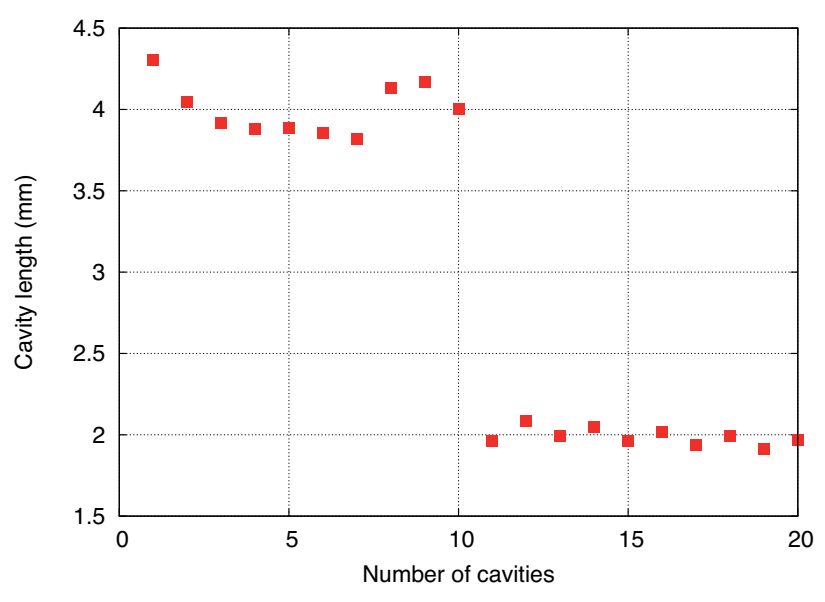

Fig. 3. The length of each resonant cavity of cascaded RE-MZM.

short electrode length is due to the large index mismatch between the electrode and the optical waveguide. The walk-off length of the current configuration $\left(N_{m}\right.$ $=3.086$ and $N_{o}=2.15$ ) at $20 \mathrm{GHz}$ is only $8 \mathrm{~mm}$ which is less than twice the wavelength of the microwave signal $\left(\lambda_{e}=4.86 \mathrm{~mm}\right)$. Therefore, the cavity length of the single cavity RE-MZM has to be limited to $\lambda_{e}$ to avoid modulation efficiency penalty. However, when the electrode is periodically inductively loaded, the total electrode length can be extended well beyond the the walk-off length. Because of the total maximum length limit of $4 \mathrm{~cm}$, the length of each cavity has to be reduced from around $4 \mathrm{~mm}$ to $2 \mathrm{~mm}$ which is slightly less than $\lambda_{e} / 2$ when the number of cavity is larger than 10 .

Fig. 4 shows the calculated effective $V_{\pi}$ at $20 \mathrm{GHz}$ of the resulting RE-MZM as a function of the number of resonant cavities. Since the electrooptic interaction length increases with the number of resonant cavities, increasing the number of resonant cavities improves the modulation efficiency. However, there is a sharp increase of $V_{\pi}$ when the number of cavities increases from 10 to 11 . The length of each resonant cavity of 11 cavity RE-MZM is much shorter than that of the 10 cavity REMZM. Hence, the 11 cavity RE-MZM has much shorter total electrode length than the 10 cavity RE-MZM. This causes $V_{\pi}$ of 11 cavity RE-MZM higher than that of the 10 cavity RE-MZM. 


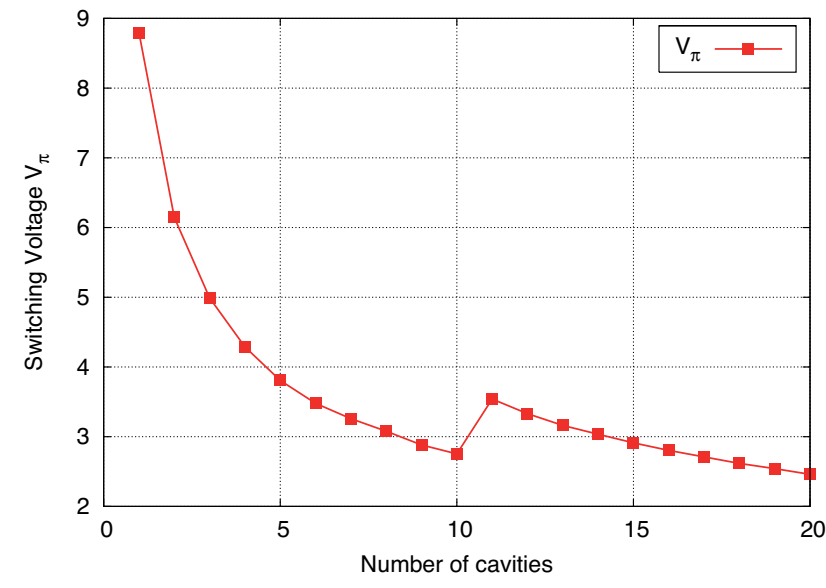

Fig. 4. Effective $V_{\pi}$ at $20 \mathrm{GHz}$ of cascaded RE-MZM with different number of cavities.

\section{PERFormance of CASCADED MUlticAVity RE-MZMS}

This section analyzes the performance of the 10 cavity RE-MZM and compares that to the single cavity REMZM. In this investigation, the inductive terminations are represented by the shorted CPW stubs. The stubs are designed in the same $\mathrm{LiNbO}_{3}$ substrate as the modulator electrode. The microwave characteristics of the stubs were calculated by the FEM simulator. The stub lengths were calculated from the equivalent inductive elements found in the previous section. When simulating the modulator response using the network model [11], the loss of the stubs was taken into account.

The frequency dependent effective $V_{\pi}$ of the 10 cavity and single cavity RE-MZMs is shown in Fig. 5. It can be seen that operation at several resonant frequencies is possible. However, the peak resonant enhancement frequency is at $20 \mathrm{GHz}$. Also shown in Fig. 5 is the effective $V_{\pi}$ calculated with the termination represented by ideal inductive elements. At $20 \mathrm{GHz}$, the $V_{\pi}$ of the single cavity RE-MZM calculated with shorted stubs and ideal inductive terminations are very close to each other. However, the calculated $V_{\pi}$ using the shorted stubs is $18 \%$ higher than $V_{\pi}$ obtained with ideal inductive terminations. This reduction of the modulation efficiency is caused by the RF loss of the stubs. The total RF loss of the stubs in the single cavity RE-MZM is small and it can be ignored. Whereas, the 10 cavity RE-MZM has a large number of stubs; hence, the RF loss due to the stubs is significant. Thus, if the terminations can be realized on a low loss microwave substrate, the degradation of modulation efficiency can be minimized.

\section{Conclusions}

In this paper, highly efficient resonantly enhanced optical modulators based on cascaded multicavity structure have been analyzed. It has been shown that with inductive terminations periodically added on the active electrode, the interaction length of the electrode can be made

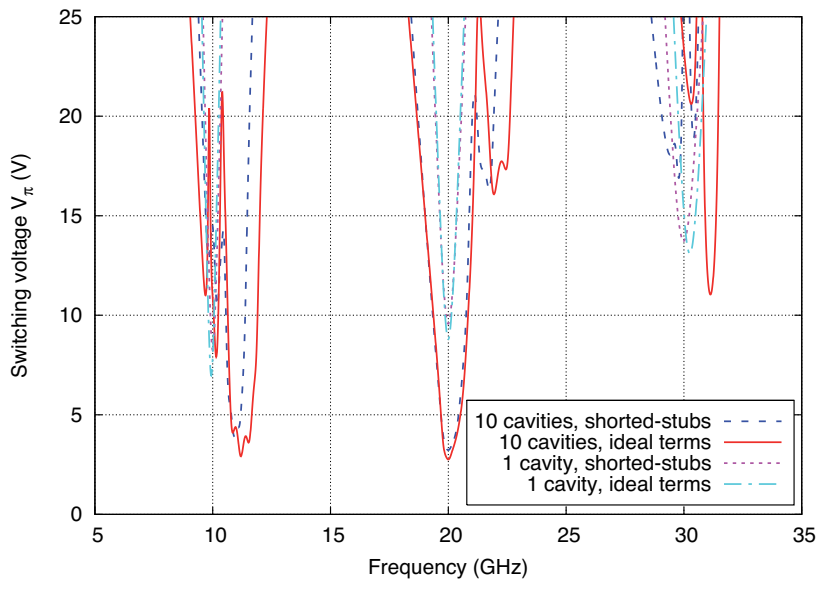

Fig. 5. Frequency dependent effective $V_{\pi}$ of a 10 cavity cascaded RE-MZM in comparison with that of a single cavity RE-MZM.

very large even with a large index mismatch between the RF electrode and optical waveguide. Hence, the modulation efficiency increases. Without the requirement of index matching, the electrode can be easily optimized to provide high inherent electrooptic interaction and low RF loss to achieve maximum modulation efficiency with resonant enhancement.

\section{REFERENCES}

[1] A. Nirmalathas, D. Novak, C. Lim, R. Waterhouse, and D. Castleford, "Fiber networks for wireless applications," in LEOS 2000 Annual Meeting, Conference Proceedings, vol. 1, 2000, pp. 3536.

[2] A. Lindsay, G. Knight, and S. Winnall, "Photonic mixers for wide bandwidth RF receiver applications," IEEE Trans. Microwave Theory Tech., vol. 43, no. 9, pp. 2311-2317, Sept. 1995.

[3] T. Kawanishi, S. Oikawa, K. Higuma, Y. Matsuo, and M. Izutsu, "Resonant electrode structures for band-operation-type optical modulators," Proceedings of SPIE, vol. 4872, pp. 1-10, 2002.

[4] R. Krähenbühl and M. Howerton, "Investigations on short-pathlength high-speed optical modulators in $\mathrm{LiNbO}_{3}$ with resonanttype electrodes," J. Lightwave Technol., vol. 19, no. 9, pp. 12871297, Sept. 2001

[5] T. G. Nguyen, A. Mitchell, and Y. S. Visagathilagar, "Practical demonstration of a highly efficient resonantly-enhanced MachZehnder modulator at $1.8 \mathrm{GHz}$," IEEE Photon. Technol. Lett., no. 3, Feb. 2006.

[6] - - , "Investigation of resonantly enhanced modulators on $\mathrm{LiNbO}_{3}$ using FEM and numerical optimization technique," $J$. Lightwave Technol., vol. 22, no. 2, pp. 526-533, Feb. 2004.

[7] T. Kawanishi, S. Oikawa, K. Higuma, Y. Matsuo, and M. Izutsu, " $\mathrm{LiNbO}_{3}$ resonant-type optical rnodulator with double-stub structure," Electron. Lett., vol. 37, no. 20, pp. 1244-1246, Sept. 2001.

[8] Y. S. Visagathilagar, T. G. Nguyen, and A. Mitchell, "Numerical analysis of a $20 \mathrm{GHz}$ cascaded resonantly enhanced MachZehnder modulator," 2006 Asia-Pacific Microwave Photonics Conference (AP-MWP 2006), vol. Accepted for presentation, 2006.

[9] Y. Visagathilagar, A. Mitchell, and R. Waterhouse, "Fabry-Perot type resonantly enhanced Mach-Zehnder modulator," in International Topical Meeting on Microwave Photonics, MWP'99, vol. 1, 1999, pp. 17-20.

[10] A. Mitchell, "Efficient PML boundaries for anisotropic waguide simulations using the finite element method," Ph.D. dissertation, RMIT University, Melbourne, Australia, 1999.

[11] Y. S. Visagathilagar, T. G. Nguyen, A. Mitchell, and M. W. Austin, "Sytematic design approach for optimised resonantly enhanced Mach-Zhender modulators," J. Lightwave Technol., vol. 24, no. 1, pp. 555-562, Jan. 2006. 